\title{
APPROXIMATE FUNCTIONAL COMPLEXITY
}

\author{
BY R. C. BUCK ${ }^{1}$
}

Communicated by James H. Bramble, June 20, 1975

Hilbert's 13th problem dealt with the functional complexity of a specific function of three variables. One measure of the complexity of a function of $n$ variables is whether it can be represented in terms of functions of fewer variables according to a specific schema. For example, we may ask if a given function $F(x, y)$ is nomographic-i.e. can it be written in the form $f(\phi(x)+\psi(y))$, using only functions of one variable. A larger class of functions are those that can be represented as uniform limits of nomographic functions. Membership in such a class is a measure of the approximate functional complexity of a function $F$, and may be an appropriate concept in discussing computational approximation.

Some of the important questions dealing with complexity have been answered by the work of Vitushkin [8], Arnol'd [1], and Kolmogorov [5]. In the present note, we describe some new techniques for discussing approximate complexity and state several theorems which extend the scope of earlier results.

We first observe that if all the component functions used in a particular representation schema are sufficiently smooth, then the resulting class of representable functions will be solutions of one or more specific partial differential equations, which may in fact yield local characterizations for smoothly representable functions. (For example, smoothly representable functions of the form $f(\phi(x, y), \psi(y, z))$ must satisfy a fourth-order equation with 55 terms.) However, this observation does not seem to be immediately useful in treating approximate representation, nor in dealing with representation by functions required only to be continuous. (It is tempting to hope that an appropriate concept of weak solution will be useful here.)

Our results are of two types. The first is based on the study of level sets, as with much of the preceding work in complexity; excellent surveys may be found in Sprecher [7], and in [2] and [6]. Any representation schema can be regarded as a mapping diagram whose commutativity imposes necessary conditions on each component function, which in turn give rise to relations between their level sets. In some cases, this may be carried over to approximate representation. The following is typical.

AMS (MOS) subject classifications (1970). Primary 41A30; Secondary 26A72.

1 Research supported by NSF grant MPS75-06687 and ARO contract DA31$124 A$ ROD462. 
TheOREM 1. Let $F(x, y, z)$ be defined on a block $D=I_{1} \times I_{2} \times I_{3}$ in $\mathbf{R}^{3}$; choose $z_{1} \neq z_{2}$ in $I_{3}$ and define a mapping $T$ on $I=I_{1} \times I_{2}$ into $\mathbf{R}^{2}$ by

$$
T(x, y)=\left(F\left(x, y, z_{1}\right), F\left(x, y, z_{2}\right)\right) \text {. }
$$

Let $F(D)$ be the class of functions on $D$ of the form $f(\phi(x, y), z)$ where $\phi$ is continuous on I but $f$ is unrestricted. Then: (a) if $F \in F(D), T$ must be constant on a noncountable subset of $I$; (b) if $F$ lies in the uniform closure of $F(D)$, then $T$ must be constant on subsets of I of arbitrary finite cardinality.

For example, the function $x y+y z+x z$ cannot be approximated by functions in the class $F$ on any open set; the same technique used in Theorem 1 allows one to estimate the distance from this function to $F(D)$.

The second new technique is based on the use of functional inequalities, and stems from [4]. The following application to the nomographic class is typical.

THEOREM 2. Let $F(x, y)$ be positive and separately monotonic in the first quadrant. Let $I=[a, b]$ with $0<a<b$, and let $N\left(I^{2}\right)$ be the class of functions of the form $f(\phi(x)+\psi(y))$ where $\phi$ and $\psi$ are in $C[I]$, but $f$ is unrestricted. If $F$ lies in the uniform closure of $N\left(I^{2}\right)$, then for any $\epsilon>0$ there must exist $u, v, w$ with $a<u<v<w<b$, such that

$$
|F(a, v)-F(u, u)|<\epsilon, \quad|F(u, w)-F(v, v)|<\epsilon, \quad|F(a, w)-F(u, v)|<\epsilon .
$$

This can be used to show that functions such as $x^{2} y+x y^{2}$ and $x^{2}+y^{2}+$ $x y$ cannot be approximated by nomographic functions in the class $N$ on any open set in the first quadrant, and one may also obtain estimates for their distance to $N\left(I^{2}\right)$. (See [3].)

In contrast, it may be shown that any function $F(x, y)$ has the form $f(\phi(x)+2 \phi(y))$, where $\phi$ is continuous except on a countable set.

The proof of Theorem 2 uses a homotopy argument to show the existence of $u, v$, and $w$; details will appear elsewhere.

\section{REFERENCES}

1. V. I. Arnol'd, Representation of continuous functions of three variables by the superposition of continuous functions of two variables, Mat. Sb. 48 (90) (1959), 3-74; English transl., Amer. Math. Soc. Transl. (2) 28 (1963), 61-147. MR 22 \#12191; 27 \#3759.

2. - Some questions on approximation and representation of functions, Proc. Internat. Congress Math., 1958, pp. 339-348; English transl., Amer. Math. Soc. Transl. (2) 53 (1966), 192-201. MR 22 \#12192.

3. - On the representability of a function of two variables in the form $\chi[\phi(x)+\psi(y)]$, Uspehi Mat. Nauk. 12 (1957), no. 2, (74), 119-121. (Russian) MR $19,841$.

4. R. C. Buck, On approximation theory and functional equations, J. Approximation Theory 5 (1972), 228-237.

5. A. N. Kolmogorov, On the representation of continuous functions of many variables by superposition of continuous functions of one variable and addition, Dokl. Akad. Nauk SSSR 114 (1957), 953-956; English transl., Amer. Math. Soc. Transl. (2) 28 (1963), 55-59. MR 22 \#2669; 27 \#3760.

6. G. G. Lorentz, Metric entropy, widths, and superpositions of functions, Amer. 
Math. Monthly 69 (1962), 469-485. MR 25 \#5323.

7. D. A. Sprecher, Survey of solved and unsolved problems on superposition of functions, J. Approximation Theory 6 (1972), 122-134.

8. A. G. Vituskin, Estimation of the complexity of the tabulation problem, Fizmatgiz, Moscow, 1959; English transl., Theory of the transmission and processing of information, Pergamon Press, New York and London, 1961. MR 22 \#8265; 24 \#A21 87.

MATHEMATICS DEPARTMENT AND MATHEMATICS RESEARCH CENTER, UNIVERSITY OF WISCONSIN, MADISON, WISCONSIN 53706 